\title{
Caracterización de la atención de hombres ancianos por servicio de atención móvil de urgencia
}

\section{Characterization of assistance in elderly men assisted in a mobile emergency care service \\ Caracterização do atendimento de homens idosos pelo serviço móvel de urgência}

Lívia Carvalho Pereira'; Alaíde de Araújo Alencar²; Maria do Livramento Fortes Figueiredo;

Cinara Maria Feitosa Beleza ${ }^{4}$

${ }^{1}$ Mestre em Enfermagem pelo Programa de Pós-Graduação Mestrado em Enfermagem da UFPI. Enfermeira graduada pela Universidade Federal do Piaui/UFPI. Teresina, PI.

${ }^{2}$ Enfermeira graduada pela Universidade Federal do Piaui/UFPI. Teresina, PI.

${ }^{3}$ Doutora em Enfermagem pela Universidade Federal do Rio de Janeiro. Professora da Universidade Federal do Piauí da Graduação e do Programa de Mestrado em Enfermagem. Teresina, PI.

${ }^{4}$ Mestre em Enfermagem pelo Programa de Pós-Graduação Mestrado em Enfermagem da UFPI. Enfermeira graduada pela Universidade Federal do Piaui/UFPI. Teresina, PI.

Cómo citar este artículo en edición digital: Pereira, L.C., Alencar, A.A., Fortes Figueiredo, Ma L.., Feitosa Beleza, C.Ma (2015). Caracterización de la atención de hombres ancianos por servicio de atención móvil de urgência. Cultura de los Cuidados (Edición digital), 19, 41. Disponible en: http://dx.doi.org/10.14198/cuid.2015.41.15>

Correspondência: Lívia Carvalho Pereira. Endereço: Quadra 14, Bloco 9, AP 103, Conjunto Morada Nova II, Teresina-PI. Cep: 64023-208. E-mail:, tel.: (86) 9904-5887 Correo electrónico: livia.zinha@hotmail.com Recibido: 23//2014; Aceptado: 23/02/2015

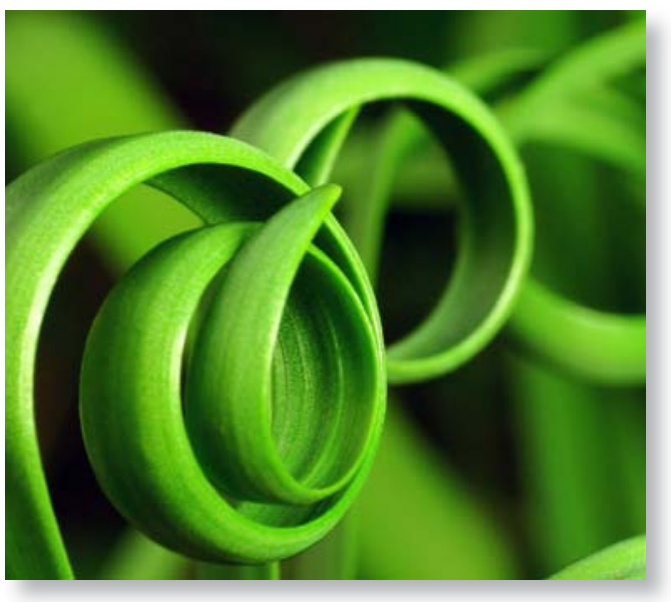

ABSTRACT

The elderly population has shown a significant increase in life expectancy in recent decades, however, they remain a population susceptible to many health hazards due to the declination of their functional physiological reserves. Coupled with this fact is the absence of public policies that prioritize the effectiveness of primary care as a gateway to the Unified Health System. This audience easily aggravates its health situation, often requiring attendance by the emergency and urgent care services. The objective of the present study was to characterize the care of elderly men by mobile emergency care service - SAMU. This is a cross-sectional, descriptive, exploratory study was developed in SAMU Teresina-PI, collection the attendance records held by SAMU between September and December 2011. The results point to a demand by the SAMU by elderly people, in the evaluated months, mainly by clinical complaints. The findings reinforce the need for more primary care coverage of health strategies that promote a better quality of life, prevention and recovery of their health.

Key words: Answering Services, Elderly, Nursing, Healthy profile. 


\section{RESUMEN}

La población de edad avanzada ha mostrado un aumento significativo en la esperanza de vida en las últimas décadas, sin embargo, se ha vuelto susceptible a muchos riesgos para la salud debido a la disminución de su reserva funcional fisiológica. Junto con este hecho es la ausencia de políticas públicas que priorizan la eficacia de la atención primaria como puerta de entrada al Sistema Único de Salud - SUS. Este público ha agravado fácilmente su estado de salud, la atención a los servicios de urgencia y emergencia con frecuencia que sea necesaria. El objetivo de este estudio fue caracterizar la atención de los hombres de edad avanzada a través del servicio de atención de emergencia móvil - SAMU. Se trata de un estudio transversal, descriptivo, exploratorio se desarrolló en SAMU Teresina-PI, se utilizó como medio de registros de asistencia de recopilación de datos llevada a cabo por el SAMU, entre septiembre y diciembre de 2011. Los resultados apuntan a una demanda SAMU por los hombres de edad avanzada en la evaluación, en especial por las quejas clínicas. Los resultados refuerzan la necesidad de mayores estrategias de cobertura de la atención primaria y de salud que promuevan una mejor calidad de vida, prevención y recuperación de la salud del público.

Palavras clave: Servicios de contestadora, Anciano, Enfermería, Perfil de Salud.

\section{RESUMO}

A população idosa tem apresentado um aumento significativo na sua expectativa de vida nas últimas décadas, contudo, a mesma apresenta-se suscetível a diversos agravos a saúde devido ao declínio de suas reservas fisiológicas funcionais. Aliada a este fato está a ausência de políticas públicas que priorizem a efetivação da atenção básica como porta de entrada ao Sistema
Único de Saúde - SUS. Este público tem agravado facilmente a sua situação de saúde, muitas vezes sendo necessário o atendimento pelos serviços de urgência e emergência. $\mathrm{O}$ objetivo do presente trabalho foi caracterizar o atendimento de homens idosos pelo serviço de atendimento móvel de urgência - SAMU. Trata-se de um estudo transversal, descritivo, exploratório que foi desenvolvido no SAMU de Teresina-PI, utilizou-se como meio de coleta de dados os registros de atendimento realizados pelo SAMU entre setembro a dezembro de 2011. Os resultados apontam para uma procura ao SAMU pelos homens idosos, nos meses avaliados, principalmente por queixas clínicas. Os achados reforçam as necessidades de maior cobertura da atenção primária e de estratégias de saúde que promovam melhor qualidade de vida, prevenção e recuperação da saúde deste público.

Palavras-chave: Serviços de atendimento, idoso, Enfermagem, Perfil de saúde.

\section{INTRODUÇÃO}

$\mathrm{O}$ atendimento pré-hospitalar no Brasil é composto por duas instâncias, fixa e móvel, apresentando enorme diversidade de serviços com distintos papéis e formas organizacionais. Tal serviço é realizado pelo Serviço de Atendimento Móvel de Urgência SAMU-192, oficializado pela Portaria no 1.864 /GM2, que institui o componente pré-hospitalar móvel da Política Nacional de Atenção às Urgências, por intermédio da sua implantação no território brasileiro (Brasil, 2006).

A regulação médica das urgências deve ser regionalizada, hierarquizada, descentralizada, pactuada, ter a participação da comunidade e atender aos princípios da integralidade, da universalidade e da equidade. Há a participação de um médico regulador que monitora o 
atendimento local via rádio, estabelecendo-se uma rede de comunicação entre a central, as ambulâncias e os serviços que recebem os pacientes. Os serviços de segurança e salvamento também devem ser orientados por esse profissional (Deslandes; Souza, 2010).

O SAMU tem como foco atender às urgências de forma integral, igualitária e com equidade, de acordo com os pressupostos do Sistema único de saúde (SUS), regulando o fluxo de paciente que é atendido desde a atenção básica até níveis de alta complexidade. Porém, observa-se a inexistência de um plano diferenciado no atendimento ao idoso. Uma das dificuldades é a falta de serviços especializados que não acompanha o crescimento dessa demanda (Mello; Moysés, 2010).

A assistência prestada pelo SAMU é executada por dois tipos de equipes, a de suporte básico e a de suporte avançado, e a atenção no ambiente pré-hospitalar é baseada na regulação médica das urgências. As equipes de suporte básico, compostas por condutores e auxiliares/técnicos de enfermagem, realizam medidas de suporte não invasivas, já as de suporte avançado, compostas por condutores, enfermeiros e médicos, executam procedimentos invasivos na assistência de urgências e emergências (Marques; Lima; Ciconet, 2011).

Observa-se no contexto atual da sociedade, uma diferenciação nos indicadores de morbimortalidade quando relacionados ao sexo feminino e masculino, a mortalidade masculina em praticamente todas as idades é mais elevada quando comparada à feminina. Com o avanço da idade, percebe-se uma maior prevalência de deficiência e incapacidade, em mulheres, o que pode ser explicado pela maior sobrevida destas (Laurenti; Jorge; Gotlieb, 2005).

Com a proposta de fortalecimento da saúde da população masculina, o Ministério da
Saúde criou a Política Nacional de Atenção Integral à Saúde do Homem (PNAISH). Esta política objetiva melhoria das condições de saúde da população masculina, contribuindo para a redução da morbidade e mortalidade dessa população, através do enfrentamento racional dos fatores de risco e mediante a facilitação ao acesso, às ações e aos serviços de assistência integral à saúde (Brasil, 2009).

Observa-se uma interface entre a PNAISH e a política nacional de atenção às urgências no que tange à saúde do homem idoso. Por exemplo, embora, como verificado nas estatísticas, reportagens, nos casos de violência no trânsito, nos quais se verifica que as mortes por essas causas são prevalentes em adultos jovens, as taxas de mortalidade apresentam-se crescentes à medida que avança a idade, Ademais, têm-se ainda a busca pelo atendimento pré-hospitalar pelo idoso devido a violências, bem como outros agravos (Gomes; Barbosa; Caldeira, 2010).

Diante desse contexto, esse estudo tem por objetivo caracterizar o atendimento de homens idosos pelo serviço de atendimento móvel de urgência - SAMU.

\section{MATERIAL E MÉTODOS}

Trata-se de um estudo transversal, descritivo, exploratório que foi desenvolvido no SAMU de Teresina-PI. Este serviço público/ municipal realiza assistência em situações de urgência clínica, traumática, psiquiátrica e obstétrica, em via pública, serviços de saúde ou domicílio, além do transporte de pacientes que necessitam de transferências interserviços de saúde.

A coleta de dados foi realizada no período de março a abril de 2012, após aprovação pelo Comitê de Ética em Pesquisa e autorização dos departamentos envolvidos no estudo. A popu- 
lação de estudo é constituída por pacientes do sexo masculino, a partir de 60 anos de idade, atendidos durante o período de setembro a dezembro de 2011, após a exclusão dos eventos obstétricos devido a tais especificidades não acometerem a população em estudo, obteve-se uma amostra de 1417 sujeitos.

Utilizou-se a ficha de ocorrência do SAMU, que trata-se de um instrumento padronizado que contem dados detalhados do paciente e da ocorrência e agravo ocorrido ao paciente, também lançou-se mão de dados de fonte secundária os quais foram coletados por meio do sistema de informações da central de regulação de urgência do SAMU de Teresina-PI, referentes às solicitações clínicas atendidas no período amostral do estudo.

Posteriormente construiu-se um formulário contendo as variáveis do estudo que incluíam dados sociodemográficos do paciente, natureza da ocorrência, sinais clínicos, condições ao chegar ao destino e procedimentos/ intervenções realizados, em seguida os mesmos foram transportados às planilhas do Programa Microsoft Excel 2010, foram realizados cálculos de frequência, médias e então foram apresentados em forma de gráficos e tabelas, e discutidos à luz da literatura estudada.

$\mathrm{O}$ estudo foi registrado e aprovado pelo Comitê de Ética e Pesquisa (CEP) da Universidade Federal do Piauí com CAAE: 0162.0.045.000-11, e atende às exigências das Diretrizes e Normas Regulamentadoras de Pesquisas Envolvendo Seres Humanos regidas pela Resolução 196/96 do Conselho Nacional de Saúde.

\section{RESULTADOS E DISCUSSÃO}

Foram coletadas informações em 14.654 fichas de ocorrência referentes aos atendimentos realizados pela equipe do SAMU de Tere- sina com saída de viatura da sede do serviço de urgência durante o período de Setembro a Dezembro de 2011. Deste total, 1.417 (9,7\%) indicavam atendimento a homens idosos. O crescente aumento no número de idosos no Brasil acentua as doenças próprias do envelhecimento, provocando aumento da carga de doenças na população, mais incapacidades e aumento do uso de serviços de saúde (Veras, 2009).

A Tabela 01 aponta a distribuição, por faixa etária, de homens idosos atendidos pelo SAMU de Teresina, no período de Setembro a Dezembro de 2011.

Tabela 01 - Distribuição de homens idosos atendidos pelo SAMU - Teresina-PI, por faixa etária, 2011. $(n=1417)$

\begin{tabular}{lrc}
\hline Idade & $\mathrm{f}$ & $\%$ \\
\hline 60 a 69 anos & 499 & 35,2 \\
70 a 79 anos & 545 & 38,5 \\
80 a 89 anos & 286 & 20,2 \\
90 a 99 anos & 82 & 5,8 \\
Igual ou maior que 100 anos & 5 & 0,3 \\
\hline Total & 1417 & 100 \\
\hline
\end{tabular}

Fonte: Pesquisa direta, 2012

Observa-se que a maioria dos homens idosos atendidos pelo SAMU de Teresina enquadra-se na faixa etária de 70 e 79 anos $(38,5 \%)$, 499 idosos atendidos tinham idade entre $60 \mathrm{e}$ $69 \operatorname{anos}(35,2 \%), 286$ homens possuíam entre 80 e 89 anos (20,2\%), 82 idosos apresentavam idade entre 90 e 99 anos (5,8\%), e apenas 5 idosos atingiam a faixa etária de igual ou maior que 100 anos (0,3\%).

Acerca dos agravos clínicos atendidos pelo SAMU de Porto Alegre, verificou- 
-se que a maior frequência de atendimentos concentrou-se na faixa etária de 41 a 60 anos $(27,54 \%)$, seguida da faixa entre 61 e 79 anos $(24,46 \%)$ e de 21 a 40 anos $(14,80 \%)$. Tais resultados assemelham-se aos achados deste estudo (Marques; Lima; Ciconet, 2011),

A prevalência de doenças crônicas apresenta-se proporcional à idade, e mais significativa ainda nos idosos. Esse fator implica em limitação das atividades diárias e contribui para a diminuição da qualidade de vida dos idosos. A limitação de atividades geralmente é maior em mulheres, enquanto que a mortalidade prevalece no grupo masculino (Laurenti; Jorge; Gotlieb, 2005).

A seguir, o Gráfico 01 dispõe da distribuição dos tipos de ocorrências que acometem os homens idosos que foram atendidos pelo SAMU de Teresina no período de Setembro a Dezembro de 2011.

Gráfico 01 - Distribuição dos tipos de ocorrências que acometem os homens idosos atendidos pelo SAMU - Teresina/PI, 2011. $(n=1417)$

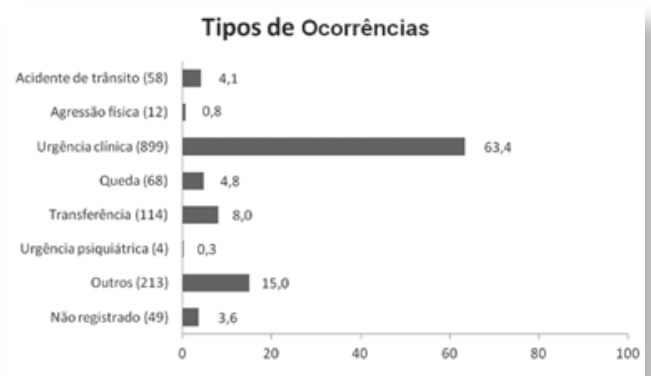

Fonte: Pesquisa direta, 2012

Diante dos diversos tipos de ocorrência atendidos diariamente pelo SAMU, no grupo estudado verifica-se a predominância de solicitações de atendimento em função de urgência clínica $(63,4 \%)$. Observou-se 114 solicitações do serviço para realização de transferências
$(8,0 \%), 68$ chamadas devido a quedas $(4,8 \%)$ e 58 atendimentos a vítimas de acidentes de trânsito $(4,1 \%)$. As agressões físicas representaram $0,8 \%$ dos casos atendidos, e as urgências psiquiátricas somaram $0,3 \%$ do total de solicitações. Foram registradas 213 ocorrências por motivos diversos aos já citados (15,0\%). Verificou-se que em 49 solicitações atendidas o tipo de ocorrência não foi registrado na ficha de ocorrência do serviço.

A prevalência de ocorrências por causas clínicas observada neste estudo, também é evidenciada em outros estudos acerca de atendimentos pelo SAMU e em diversas faixas etárias. Em estudo sobre as ocorrências atendidas pelo SAMU entre fevereiro e junho de $2006 \mathrm{em}$ um município do nordeste brasileiro, encontraram a prevalência de atendimentos por causas clínicas $(57,0 \%)$, seguido das causas externas $(32,9 \%)$ e remoções $(7,3 \%)$, sendo maior a incidência de atendimentos a idosos, que representaram também um terço dos atendimentos por causas clínicas (Cabral; Souza, 2008).

Os agravos clínicos constituem situações de alteração das condições vitais do indivíduo nas quais é necessária uma intervenção precoce e qualificada, ocasionando a procura por serviços de saúde, dentre os quais inclui-se os serviços de atendimento pré-hospitalar móvel (Marques; Lima; Ciconet, 2011). Nas situações de urgência clínica registradas no presente estudo observou principalmente agravos cardiológicos, neurológicos e metabólicos. Incluem-se nos registros: diabetes mellitus descompensada, perda da consciência, cefaleia intensa, acidente vascular cerebral (AVC) e infarto agudo do miocárdio (IAM) dentre outras situações.

As taxas de mortalidade e morbidade por AVC e IAM crescem progressivamente com o avanço da idade. A detecção precoce destes agravos além da qualidade da atenção secun- 
dária e terciária é importante por contribuir para o aumento da sobrevida destes pacientes (Luz;Junger;Cavalini, 2010).

A Tabela 02, apresentada a seguir, aponta a distribuição das variáveis analisadas no momento do atendimento realizado pelo SAMU de Teresina a homens idosos, no período de Setembro a Dezembro de 2011. Foram abordadas variáveis referentes à circulação, estado neurológico, condições de entrada no hospital e ocorrência de óbito.

Tabela 02 - Distribuição de sinais clínicos no momento do atendimento a homens idosos pela equipe do SAMU - Teresina/PI, 2011. $(n=1417)$

\begin{tabular}{|c|c|c|}
\hline Variável & $f$ & $\%$ \\
\hline \multicolumn{3}{|l|}{ CIRCULAÇĀO } \\
\hline \multicolumn{3}{|l|}{ Pulso } \\
\hline Ausente & 34 & 2,4 \\
\hline Forte & 990 & 69,9 \\
\hline Fraco & 86 & 6,1 \\
\hline NR & 307 & 21,6 \\
\hline \multicolumn{3}{|l|}{ Pressão arterial } \\
\hline Hipertenso & 384 & 27,1 \\
\hline Hipotenso & 128 & 9,0 \\
\hline Normotenso & 330 & 23,3 \\
\hline NR & 575 & 40,6 \\
\hline \multicolumn{3}{|l|}{ Sangramento } \\
\hline Presente & 107 & 7,6 \\
\hline Ausente & 828 & 58,4 \\
\hline NR & 482 & 34,0 \\
\hline \multicolumn{3}{|l|}{ ESTADO NEUROLÓGICO } \\
\hline \multicolumn{3}{|l|}{ Nivel de consciência } \\
\hline Alerta & 908 & 64,1 \\
\hline Responde a comando & 133 & 9,4 \\
\hline Responde a dor & 66 & 4,7 \\
\hline Não responde & 83 & 5,8 \\
\hline NR & 227 & 16,0 \\
\hline \multicolumn{3}{|l|}{ Pupilas } \\
\hline Isocóricas & 1034 & 72,9 \\
\hline Anisocóricas & 25 & 1,8 \\
\hline NR & 358 & 25,3 \\
\hline \multicolumn{3}{|l|}{ Fala } \\
\hline Normal & 810 & 57,2 \\
\hline Confusa & 145 & 10,2 \\
\hline Nenhuma & 186 & 13,1 \\
\hline NR & 276 & 19,5 \\
\hline CONDIC̨ÃO DE & NO & \\
\hline HOSPITAL & 62 & 4,4 \\
\hline Melhor & 8 & 0,6 \\
\hline Pior & 584 & 41,2 \\
\hline Inalterado & 763 & 53,8 \\
\hline NR & & \\
\hline \multicolumn{3}{|l|}{ OCORRÊNCIA DE ÓBITO } \\
\hline Antes do socorro & 39 & 2,7 \\
\hline Antes do transporte & 4 & 0,3 \\
\hline Durante o transporte & 4 & 0,3 \\
\hline NR ou não houve óbito & 1370 & 96,7 \\
\hline
\end{tabular}

Legenda: NR - Não registrado na ficha de ocorrência FONTE: Pesquisa direta - 2012
Na categoria Circulação, observa-se que os homens idosos, em geral, apresentam pulso forte $(69,9 \%)$, ausência de sangramento $(58,4 \%)$ e até pico hipertensivo $(27,1 \%)$ durante $o$ atendimento. Verifica-se que, na maioria dos atendimentos (40,6\%), não foi registrada na ficha de ocorrência a pressão arterial do paciente. A identificação do estado neurológico dos pacientes atendidos permite verificar que a maioria apresenta-se consciente e alerta $(64,1 \%)$, com pupilas isocóricas $(72,9 \%)$ e fala normal $(57,2 \%)$.

As condições de circulação e estado neurológico no momento do atendimento permitem traçar o perfil dos atendimentos ao grupo estudado, quanto a dados referentes a pressão arterial, pulsação, sangramento, nível de consciência, condições de fala e pupilas. Além de orientar a equipe do serviço pré-hospitalar móvel, tais informações servem de base para o atendimento hospitalar. Entretanto este dado, em muitos casos, não chega a ser registrado na ficha de atendimento, dificultando a identificação das condições gerais do paciente e do trabalho da equipe.

Quanto à condição de entrada do paciente no hospital de destino, em mais da metade dos casos $(53,8 \%)$ este dado não foi registrado na ficha de atendimento. Nas fichas em que há esse registro, observa-se que na maioria dos atendimentos $(41,2 \%)$ não houve alteração do estado de saúde do paciente até a entrada no hospital de destino. A condição de entrada do paciente no hospital de destino configura importante instrumento para avaliar a evolução do transportado durante todo o percurso e mediante o atendimento da equipe do SAMU. A subnotificação observada na maior parte dos casos compromete uma avaliação sobre tal informação.

Quanto a ocorrência de óbito, foram registrados 39 óbitos antes do atendimento pela 
equipe do SAMU, 4 óbitos antes do transporte e 4 durante o transporte. Contudo, 96,7\% das fichas de ocorrência não informavam sobre a ocorrência ou não de óbito da vítima atendida. Ressalta-se que no instrumento utilizado pelo SAMU para registro das informações, quanto a ocorrência de óbito, dispõe-se das seguintes alternativas: antes do socorro, antes do transporte e durante o transporte, porém não há alternativa sobre a não ocorrência de óbito. Desta forma, nas fichas em que este campo não foi preenchido, não há como inferir sobre a influência da subnotificação de tal dado. Tal subnotificação, em que os desfechos não foram registrados nas fichas de atendimento, também foi encontrada em outros estudos (Marques; Lima; Ciconet, 2011).

O panorama atual do Brasil quanto a morbimortalidade masculina assemelha-se ao padrão de outros países, em que os coeficientes de mortalidade masculina apresenta-se cerca de 50\% maiores em relação ao sexo oposto. Segundo os autores, dentre as causas desse dado, sobressaem mortes por doenças do aparelho circulatório seguidas por aquelas relativas a acidentes e violências (Laurenti; Jorge; Gotlieb,2005).

Gráfico 02 - Frequência dos procedimentos realizados durante atendimento a homens idosos pelo SAMU de Teresina.

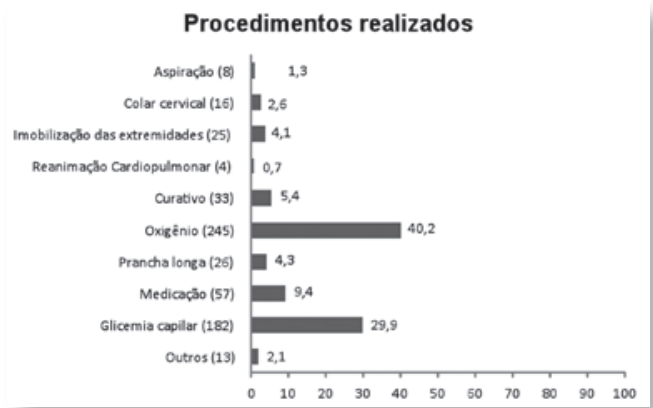

Fonte: Pesquisa direta, 2012.
Embora durante o atendimento ao homem idoso em diferentes situações exija a realização de procedimentos diversos, este dado muitas vezes deixa de ser notificado. Foram registrados 609 procedimentos realizados no total de ocorrências atendidas, entretanto, em muitas fichas de atendimento esse dado não foi informado. Verifica-se que os principais procedimentos realizados envolvem principalmente a administração de oxigênio (40,2\%) e o teste de glicemia capilar $(29,9 \%)$, e incluem ainda a administração de medicação $(9,4 \%)$, realização de curativos $(5,4 \%)$, uso de prancha longa(4,3\%), imobilização de extremidades $(4,1 \%)$, colar cervical $(2,6 \%)$, aspiração $(1,3 \%)$, e reanimação cardiopulmonar (0,7\%), dentre outros procedimentos não especificados $(2,1 \%)$.

Verificou-se em estudo realizado no SAMU avançado de um município da região noroeste do Estado do Rio Grande do Sul, observou-se que dentre os procedimentos mais realizados destacam-se a oximetria de pulso (33\%), administração de oxigênio (29\%), medicação endovenosa (12\%), e outros tipos de procedimentos diversos (19\%) (Moi; Pettenon, 2011). Tais resultados assemelham-se aos achados do presente estudo.

Observa-se que o envelhecimento populacional aliado à prevalência de doenças crônicas crescente conforme a idade têm sido refletida nos atendimentos a idosos pela equipe do SAMU. Além disto, deve ser considerado o fato de que os homens ainda se mostram resistentes a buscar os serviços de saúde, e que diversos agravos motivadores das ocorrências citadas poderiam ser evitados com ações da atenção básica. Destaca-se a importância de políticas públicas voltadas a homens e idosos, bem como a real efetivação das mesmas para alcançar uma redução da ocorrência de agra- 
vos, melhor qualidade de vida e maior sobrevida dos homens idosos.

\section{CONCLUSÃO}

A saúde do homem idoso constitui uma área ainda pouco explorada, porém em fase de expansão. A produção científica acerca do atendimento pré-hospitalar destinado a essa população é ainda mais escassa, demonstrando a necessidade de pesquisas e produção de conhecimento nesta temática.

Diante dos dados coletados, observa-se que as solicitações de atendimento a homens idosos pelo SAMU-Teresina representaram 9,7\% do total de atendimentos realizados por este serviço. A análise das categorias estudadas permite verificar que diversas variáveis não são registradas de maneira adequada, comprometendo a análise e avaliação de alguns itens. Quanto à idade verificou-se a maioria dos homens idosos atendidos pelo SAMU de Teresina enquadra-se na faixa etária de 70 e 79 anos $(38,5 \%)$.

O estado neurológico dos pacientes atendidos permite verificar que a maioria apresentava-se consciente e alerta $(64,1 \%)$. Quanto à circulação, prevaleceu a pulsação forte $(69,9 \%)$, pico hipertensivo $(27,1 \%)$ e ausência de sangramento (58,4\%). Em 41,2\% dos casos o estado de saúde do paciente permaneceu inalterado ate a chegada ao hospital. Foram registrados 47 óbitos (3,3\%), sendo a maioria de ocorrência antes da chegada do socorro pela equipe do SAMU. Dentre os procedimentos realizados os mais citados foram administração de oxigênio (40,2\%), teste de glicemia capilar $(29,9 \%)$ e administração de medicação $(9,4 \%)$.

As principais causas de solicitação de atendimento a homens idosos foram as urgências clínicas, ressaltando o avanço das taxas de morbimortalidade por AVC e IAM, dentre outros, paralelamente ao avanço da idade. Tal fato implica na importância da atenção primária em saúde a fim de evitar e reduzir os riscos de tais agravos, bem como da atenção secundária na melhoria da qualidade vida destes pacientes.

Dessa maneira foi possível ao longo desse estudo obter conhecimentos acerca do trabalho desenvolvido pela equipe do SAMU, já que este funciona como um elo entre os diferentes níveis de assistência da saúde e compreender a situação do homem idoso no panorama de atendimento pré-hospitalar móvel contribuindo desse modo na promoção de políticas públicas voltadas a sua assistência.

\section{REFERÊNCIAS}

- BRASIL. (2006). Ministério da Saúde. Secretaria de Atenção à Saúde. Política nacional de atenção às urgências / Ministério da Saúde. 3ed. ampl. Brasília: Editora do Ministério da Saúde.

- BRASIL. (2009). Ministério da Saúde. Secretaria de Atenção à Saúde. Política nacional de atenção integral à saúde do homem. Brasília: Editora do Ministério da Saúde.

- Cabral, A.P.S. y Souza, W.V. (2008). Serviço de Atendimento Móvel de Urgência (SAMU): análise da demanda e sua distribuição espacial em uma cidade do Nordeste brasileiro. Rev. Bras. Epidemiol, 11(4), 530-40.

- Chizzotti, A. (2001).Pesquisa em Ciências Humanas e Sociais. 5.ed. São Paulo: Cortez.

- Ciconet, R.M., Marques, G.Q. y Lima, M.A.D.S. (2008). Educação em serviço para profissionais de saúde do Serviço de Atendimento Móvel de Urgência (SAMU): relato da experiência de Porto Alegre-RS. Interface - Comunicação, Saúde, Educaçã,. 12(26), 659-666.

- Deslandes, S.F. y Souza, E. (2010). Atendimento pré-hospitalar ao idoso vítima de violência em cinco capitais brasileiras. Ciênc. Saúde coletiva, 15(6), 2775-2786 . 
- Gomes, L.M.X., Barbosa, T.L.A. y Caldeira, A.P. (2010). Mortalidade por causas externas em idosos em Minas Gerais, Brasil. Esc. Anna Nery Rev. Enf, 14(4), 779-786.

- IBGE. (2010). Instituto Brasileiro de Geografia e Estatística. Disponível em: <http://www.ibge.gov.br> Acessado em: 12 de abril de 2011.Síntese de Indicadores Sociais. Disponível em: http://www.ibge.gov.br/ home/presidencia/noticias/noticia_visualiza.php?id_ noticia=1717\&id_pagina=1> Acesso em: 10 de abril de 2011.

- Laurenti, R., Jorge, M.H.P.M. y Gotlieb, S.L.D. (2005). Perfil epidemiológico da morbi-mortalidade masculina. Ciênc. saúde coletiva, 10(1), 35-46.

- Leopardi, M.T. (2002). Metodologia da pesquisa na saúde. 2.ed. Florianópolis (SC): UFSC.

- Luz, C.C., Junger, W.L. y Cavalini, L.T. (2010). Análise da atenção pré-hospitalar ao acidente vascular cerebral e ao infarto agudo do miocárdio na população idosa de Minas Gerais. Rev. Assoc. Med. Bras, 56(4), 452-457 . Disponível em:<http://www.scielo.br/scielo. php?script=sci_arttext\&pid=S01044230201000040001 9\&lng=en\&nrm=iso $>$. ISSN 0104-4230. http://dx.doi. org/10.1590/S0104-42302010000400019.

- Marconi, M.A. y Lakatos, E.M. (2003). Fundamentos de metodologia científica. 5.ed. São Paulo: Atlas.

- Marques, G.Q. , Lima, M.A.D.S. y Ciconet, R.M.. (2011). Agravos clínicos atendidos pelo Serviço de Atendimento Móvel de Urgência (SAMU) de Porto Alegre - RS. Acta paul. Enferm, 24(2),185-191.

- Mello, A.L.S.F. y Moyses, S.J. (2010). Análise diagnóstica do atendimento pré-hospitalar para acidentes e violências contra idosos em Curitiba (PR, Brasil). Ciênc. saúde coletiva, 15(6), 2709-2718.

- Moi, E.C.y Pettenon, M.K. (2011). Perfil de atendimentos realizados pelo serviço de atendimento móvel de Urgência- SAMU. Defesa Trabalho de Conclusão de Curso. UNIJUÍ - Universidade Regional do Noroeste do Estado do Rio, Rio de Janeiro.

- Veras, R.(2009). Envelhecimento populacional contemporâneo: demandas, desafios e inovações. Rev. Saúde Pública, 43(3),548-544.

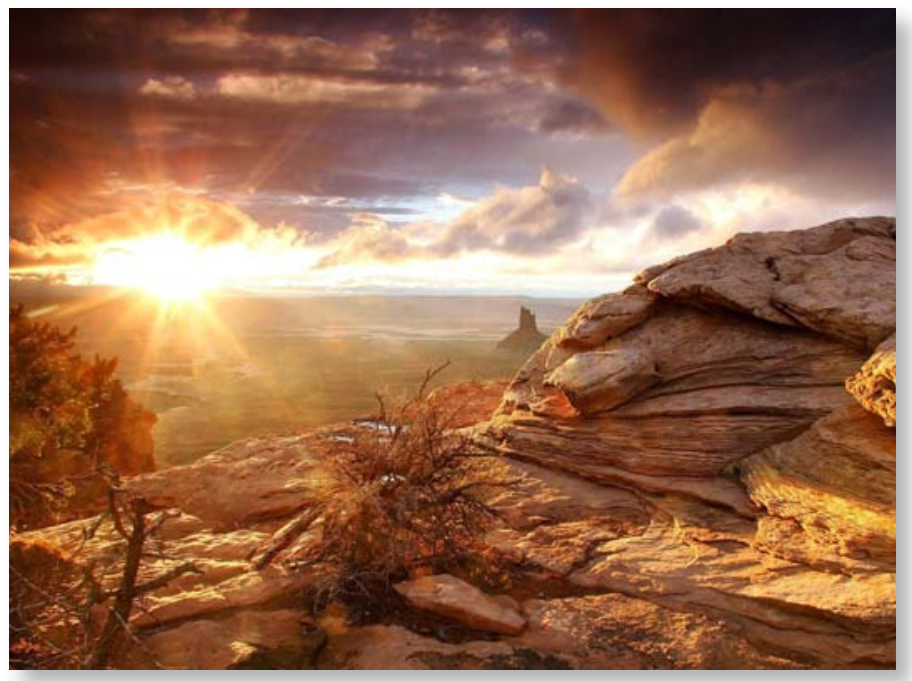

